\title{
Activismo, medios y tecnologías. Un cuestionamiento del fetichismo digital en los movimentos sociales contemporáneos
}

\section{Alejandro Barranquero ${ }^{1}$}

\section{DOI: 10.5294/pacla.2021.24.3.9}

Para citar esta reseña / To cite this book review / Para citar esta resenha Barranquero, A. (2021). Activismo, medios y tecnologías. Un cuestionamiento del fetichismo digital en los movimentos sociales contemporáneos. Palabra Clave, 24(3), e2439. DOI: https://doi.org/10.5294/pacla.2021.24.3.9

Reseña de los libros:

Treré, E. (2019). Hybrid media activism. Ecologies, imaginaries, algorithms. New York: Routledge.

Treré, E. (2020). Activismo mediático híbrido. Ecologías, imaginarios, algoritmos. Bogotá: FES.

Los estudios sobre movimientos sociales están hoy viviendo una época de esplendor que acompaña a ciclos contenciosos como las protestas antiglobalización de finales de los años noventa, los movimientos de las plazas desde 2010 -la Primavera Árabe, el 15M, Occupy, etc.- o, más recientemente, el repunte del movimiento feminista, ecologista o en defensa de las pensiones. Más allá de las diferencias existentes entre los mismos, todas las movilizaciones han tenido como común denominador un uso acentuado, constante y creativo de los medios y las tecnologías a su alcance, en especial con objeto de difundir información autónoma, captar a nuevos participantes o interconectarse en redes de activismo global. La apropiación ciudadana de estas tecnologías de la información y la comunicación (TIC) ha provoca-

1 https://orcid.org/0000-0002-9264-9389. Universidad Carlos III de Madrid, España. abarranq@hum.uc3m.es 
do que el estudio de la acción colectiva adquiera con el tiempo una orientación cada vez más tecnológica y comunicacional. En este sentido, se han multiplicado las investigaciones que se preguntan por el poder de las web, las redes sociales o las tecnologías móviles como un factor central para la creación de marcos autónomos y repertorios de protesta con fines de justicia y transformación social.

Es en este escenario de revitalización y "tecnologización” de los movimientos sociales donde sitúa la obra de un investigador emergente que lleva un tiempo examinando la relación entre activismo, medios y tecnologías. Pese a su juventud, Emiliano Treré ha plasmado en su Hybrid media activism una trayectoria académica ya extensa a lo largo de tres contextos geográficos: Italia, México y España, en los que el papel de las tecnologías ha resultado clave para la conformación de distintas movilizaciones. El recorrido por estos países le sirve como pretexto para resumir los principales aportes académicos de un campo innovador, como es el de la comunicación y los movimientos, que, si bien emerge en los años ochenta, con aportes como los de Todd Gitlin o William A. Gamson, vive en la actualidad su época de esplendor definitivo con la expansión del activismo digital. Asimismo, las páginas del libro introducen al lector en un conjunto de innovadores conceptos que ayudan a desentrañar la compleja relación entre medios y movimientos: desde las nociones de "imaginarios tecnológicos" y "prácticas mediáticas" a las de "tecnopolítica" o "activismo" y "justicia de datos".

Afincado hoy en la Universidad de Cardiff, la carrera intelectual y activista de este investigador transitó durante años por unos países que constituyen las principales paradas de un recorrido teórico y muy experiencial por distintos ciclos de protesta. En primer lugar, su Italia natal está representada por el movimiento juvenil Onda Anómala, que emergió en 2008 como una reacción a la privatización de la universidad pública y a los recortes presupuestarios en el campo de la educación. A la postre, este sería uno de distintos gérmenes tras la conformación del movimiento Cinco Estrellas (Cinque Stelle), fundado en 2009 por el cómico Beppe Grillo y cuya proyección definitiva como partido político tuvo mucho que ver con el fino olfato comunicativo de Gianroberto Casaleggio, una figura un tanto desconocida 
que es analizada en el libro. En segundo lugar, Treré se detiene en México para estudiar el \#Yosoy132, un movimiento estudiantil caracterizado por su crítica a la concentración del sistema mediático mexicano y que surgió el año 2012 en el contexto de las elecciones presidenciales que auparon al candidato del Partido Revolucionario Institucional (PRI), Enrique Peña Nieto. Por último, sus reflexiones aterrizan en España para analizar los diversos núcleos activistas que confluyeron en el $15 \mathrm{M}$, o movimiento de los indignados, que el investigador relaciona con ciclos de movilizaciones anteriores, como el 13M o el V de Vivienda y con su réplica en la Plataforma de Afectados por la Hipoteca (PAH).

A nivel teórico, el punto de partida del libro se sitúa en la "ecología de los medios”, una tradición investigadora, anticipada por clásicos como Marshall McLuhan, que en tiempos recientes está influyendo en la visión de pensadores de la talla de Nick Couldry, Andrew Chadwick o David L. Altheide. Dicho marco le permite indagar en la propia historia reciente del campo para desvelar un camino de aciertos, pero también de innumerables errores. Uno de los errores más comunes es el tecnodeterminismo que se vislumbra tras las investigaciones que glorifican la tecnología como una especie de "varita mágica" o como el desencadenante principal de la Primavera Árabe, el 15M o el movimiento Occupy. En este libro, la relación entre medios y tecnología es, en cambio, abordada desde una perspectiva mucho más matizada que ayuda a Treré a descubrir vínculos históricos entre distintos movimientos sociales o entre las múltiples prácticas a las que está ligada la socialización de las tecnologías. En esta línea, el autor traslada la teoría de los "sistemas mediáticos híbridos" (hybrid media systems) de Andrew Chadwick a la reflexión de los movimientos sociales, un aporte que le ayuda a comprender cómo los distintos medios y plataformas se intersectan hoy en un sistema híbrido de ecologías complejas que determina percepciones, usos y apropiaciones.

Uno de los grandes valores del libro es precisamente su capacidad para la contextualización histórica de cada práctica activista. Con este afán, los primeros estudios citados se corresponden con un momento en el que teóricos como Manuel Castells o Howard Rheingold se fascinaron por la 
capacidad de las tecnologías para romper el monopolio informativo o para coordinar acciones de protesta a escala global. Superada esta fase de tecnofascinación, Treré se sitúa en una posición de enorme cautela, que incluso avanza en la crítica, en uno de los intentos más agudos publicados en los últimos años, con el objeto de deconstruir todos los mitos tecnológicos asociados a las mal denominadas "redes sociales" o, más recientemente, a los macrodatos (big data). Por ejemplo, en el caso del 15M, el libro revela cómo los imaginarios tecnológicos de académicos o activistas enfatizaron en exceso en los valores "democráticos" de la red, como si esta fuese sinónimo de "horizontalidad, descentralización, ausencia de liderazgo, apertura, inclusividad y transparencia” (p. 152). Y, más allá de que dichos imaginarios condujeran a vislumbrar un nuevo horizonte de posibilidades para el activismo, la perspectiva de Treré abandona la "obsesión” por los efectos mediáticos, para adentrarse en una pregunta mucho más antropológica acerca de las prácticas ciudadanas o aquello que las personas hacen y construyen con los medios.

La tríada de conceptos que aparecen en el título, "ecologías”, "imaginarios" y "algoritmos" indica también las dimensiones centrales de su toma de partido: el concepto de ecologías le ayuda a entender de manera holística la naturaleza compleja, híbrida y multidimensional de la relación entre medios y movimientos, mientras que el de imaginarios aborda la construcción de identidades colectivas, así como un conjunto de mitos relacionados con la percepción de la técnica: el énfasis en la novedad, la transparencia, la horizontalidad, etc. Por último, los apartados finales del libro se acercan al concepto de algoritmos, como una noción en la que las posibilidades de lo tecnológico son contempladas en un escenario más complejo en el que los usos preprogramados de las tecnologías conviven con determinantes comerciales y con un enfoque material que desmonta el imaginario de la supuesta "inmaterialidad" de las tecnologías de la información.

Las reflexiones de Treré están llamadas a trascender el campo más específico de medios y movimientos sociales, dado que tienen un enorme potencial para complejizar incluso la propia teoría de la comunicación. En la línea de los maestros que dialogan en las páginas del libro (Armand 
Mattelart, Evgeny Morozov, Vincent Mosco, etc.), el trabajo profundiza en una comprensión antropológica de los medios que invita a no recaer una y otra vez en los errores del pasado: el retorno a las desgastadas teorías del "difusionismo" y su creencia en el poder mágico de las tecnologías; el mediacentrismo, o la fascinación por el soporte y no por los procesos y prácticas comunicacionales; $\mathrm{o}$, más recientemente, un retorno a la investigación cuantitativa, que difícilmente capta la sustancia que subyace a los imaginarios y sus prácticas.

Frente a la miopía del ciberfetichismo y la sublimación tecnológica, Treré critica una serie de falacias características tanto de la teoría de la comunicación como del propio estudio de la comunicación en los movimientos sociales. En particular, nos interesa reseñar lo que el italiano denomina la falacia de "un solo medio" (one-medium fallacy), o la tendencia de tantos estudios a aislar y a estudiar por separado una sola red social, medio o tecnología, pues, a la postre, así se ignoran las múltiples interrelaciones que distintos soportes mantienen en los complejos ecosistemas contemporáneos. Este reduccionismo está conectado a la falacia del "dualismo espacial” (spacial dualism), tendencia a tratar de manera diferenciada el mundo virtual y la comunicación presencial, como si no existiesen determinantes materiales o comerciales, que tantas veces frenan el fluido libre e igualitario de la información.

Al manejar distintas lenguas (italiano, inglés, español), la obra de Treré es enriquecedora, por cuanto proyecta un diálogo cada vez más necesario entre autores de diferentes latitudes y, en particular, entre la academia anglosajona y los estudios del Sur Global, en el que podemos enmarcar tanto a Europa del sur (España e Italia) como a Latinoamérica. Por ejemplo, en el texto se percibe la influencia tanto del hispano-colombiano Jesús Martín-Barbero como del británico Nick Couldry. La idea de "mediaciones" del primero le ayuda a proyectar una mirada histórica a los procesos de recepción y a la capacidad de agencia tecnológica que tienen los ciudadanos frente a los procesos de dominación y hegemonía (p. 206), mientras que la de "mediatizaciones" da cuenta del carácter ambivalente de los medios en la modernidad, por cuanto su lógica determina la mediatización de las ins- 
tituciones sociales, al tiempo que facilita una redistribución del poder entre la política institucional y la ciudadana (pp. 36-37).

Por último, en muchas páginas del libro se hace patente una crítica visceral al positivismo rampante que hoy domina en el ámbito de las ciencias sociales y comunicacionales, que las conduce a caer periódicamente en separaciones artificiales entre hechos y valores, entre teorías y prácticas, o que incluso las lleva a un estudio fragmentado de "medios viejos" frente a "nuevos" o de prácticas culturales frente a los estudios de economía política en los que toda cultura y toda comunicación se insertan. En este sentido, Treré se detiene a criticar la comodidad de la que hacen gala tantos estudios del campo cuando se limitan a analizar solo la realidad visible -o frontstage- de redes sociales como Facebook o Twitter, lo que les excusa para adentrarse en la realidad más invisible y compleja de redes que permanecen en el backstage, como WhatsApp o Telegram. Frente a los miopes acercamientos del cuantitativismo ingenuo, el libro de Treré supone uno de los acercamientos más lúcidos, integrales y cualitativos a lo evidente, pero, sobre todo, a lo implícito y no evidente en las prácticas activistas. En esta línea, su Hybrid media activism está destinado a convertirse en un clásico de los estudios del área, por cuanto ayuda a desmontar los ideales de autonomía tecnológica tantas veces asociados a Internet y, en especial, porque su acercamiento antropológico a medios y tecnologías consigue transitar, adaptando palabras de Adrienne Russell, desde las lógicas a las sensibilidades y desde los análisis estáticos, parciales y monomediáticos hacia la hibridación, la apertura y la sorpresa (p. 36). 